\title{
A Traffic Aware and Superframe Adjustment MAC Protocol for Wireless Sensor Networks
}

\author{
Seong Cheol Kim ${ }^{1}$, Hye Yun Kim ${ }^{2}$ \\ Department of Convergence Electronics, SangMyung University, Korea, sckim@smu.ac.kr \\ Department of System Semiconductor Engineering, SangMyung University, Korea, hykim@ @mu.ac.kr
}

\begin{abstract}
As applications using WSNs spread, demands for various packet transmissions are increasing. But sensors in WSNs operate by batteries, an efficient energy control mechanism is required due to the limited available energy. It is important to reduce idle listening and packet collisions to prevent unnecessary sensor energy consumption. In this paper, we present a traffic-aware and super-frame adjustment MAC protocol for WSNs which we call TASA-MAC. The TASA-MAC protocol has a slot reservation and extending frame function according to the amount of transmitted traffic. These features make TASA-MAC suitable for time critical applications. The results show that TASA-MAC protocol can reduce the packet transmission delay and prolong the network lifetime in comparison to existing related MAC protocols.
\end{abstract}

Key words : MAC Protocol, WSNs, Energy Efficiency, Delay, Duty Cycling

\section{INTRODUCTION}

Due to the development of sensor technology such as electronic technology, the application fields of wireless sensor networks are getting wider. In particular, in recent years, interest in the WBSN field, in which various indicators of the human body are measured, and when an emergency such as cardiac arrest occurs, transmits the measured information to the medical staff within a limited time is increasing. In general, WSNs are composed of battery-operated sensors that are difficult to replace. Therefore, in order to extend the life of WSNs, it is necessary to reduce the energy consumption of these sensors [9][10][11]. The main causes of energy consumption of the sensor in the MAC layer are packet transmission and reception, and idle listening. [1][2][3][4]. A duty-cycle mechanism is used to reduce energy consumption due to idle listening [6][7]. In the duty-cycle mechanism, nodes alternate between power ON and OFF states. Nodes can participate in packet transmission/reception only in the power ON state. Therefore, to reduce energy consumption, nodes should stay in the OFF state as much as possible. However, in the OFF state, packet transmission and reception are impossible, so the overall transmission delay increases. Many research works such as Routing Enhanced MAC (RMAC) [3], Demand Wakeup MAC (DW-MAC) [4] and Synchronous duty-cycle MAC (SR-MAC) [5] have been proposed to solve this problem. In this paper, we design a Traffic Aware and Super-frame Adjustment MAC Protocol (TASA-MAC) for WSNs. The paper is organized as follows. Section 2 describes the design of TASA-MAC, and Section 3 shows the performance of TASA-MAC. Finally, we draw conclusion in Section 4.

\section{DESIGN OF THE TASA-MAC PROTOCOL}

The TASA-MAC is a synchronous and contention-based duty-cycle MAC protocol. So sensor nodes in the network need to be synchronized each other for converging the wakeup and the sleep time. And the TASA-MAC protocol has a slot reservation mechanism in order to communicate with neighboring nodes in the Sleep period of one operation period. To this end, the Data period and the Sleep period are composed of the same number of slots. The TASA-MAC protocol is based on the SR-MAC protocol operation proposed in [5]. Figure 1 shows the operation of the SR-MAC protocol. As shown in the figure, the node that transmitted the control frame in the second slot of the Sleep period transmits its data in the second slot of the Sleep period. That is, the second slot of the sleep period is reserved for node $\mathrm{S}$.

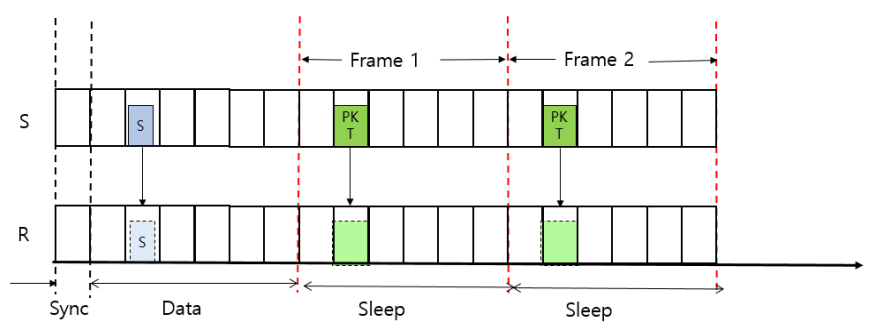

Figure 1: SR-MAC Protocol scheduling for packet transmission.

The biggest advantage of the SR-MAC protocol is that packets are transmitted without collision in the sleep period. This is because a node that wants to transmit a packet in the Data period uses a mechanism that can reserve a slot. However, if the first control frame transmitted in the Data period is made at the end of the Data period, packets 
transmitted in all Sleep periods are made in the latter part of the period. Moreover, if the number of control frames transmitted in the Data period is small, it causes waste of many slots in the Sleep period. To solve these problems, the TASA-MAC protocol was proposed in this paper. In TASA-MAC protocol an entire operation period is divided into four parts; Sync1 period, Data period, Sync2 period, and Sleep period. All nodes wake up simultaneously at the SYNC1 period to synchronize with neighbor sensor nodes as SYNC period in normal WSNs. During the Data period the senders send control frame to their neighbor nodes to participate in packet transmission. Here we call the control frame as the slot reservation and operational cycle expansion information-informed frame, REF (slot Reservation and cycle Expansion Frame). By using the reservation mechanism through this control frame, a node can transmit its own data packet without collision. In addition to information as in RTS/CTS, the REF frame includes the number of slots consists of a frame. Furthermore, the control packets include the information of the number of packets the node wants to send. Using these information, a sink node can decide whether to extend the operational cycle or not. In TASA-MAC, Data and Sleep periods are divided into M slots. But the size of the M may be changed according to the pattern of the receiving packets. And the Sleep period may compose of several frames made of $\mathrm{M}$ slots. The number of frames in a Sleep period depends on the traffics from sender nodes. In other words, the TASA-MAC adopts flexible operational cycle according to data traffic. The node that first sent its control frame in the Data period can transmit data in the first slot of all frames in the Sleep period. All other nodes other than the node that transmitted its own control frame in the Data period remain off in the Sleep period to reduce energy consumption. The Sync2 period is necessary for all nodes to obtain slot reservation and operation cycle extension information from the sink node. To do this, all nodes must wake up in Sync2 period. The advantages of TASA-MAC protocol are as follows: The node that transmits the control frame for the first time in the Data period transmits its own packet in the first slot of all frames in the Sleep period. All slots after the last control frame transmitted in the Data period are deleted from all frames in the Sleep period. Figure 2 shows an example of the operation of the TASA-MAC protocol. As you can see in the figure, nodes A and B are trying to transmit data. Since the original data period consists of 6 slots, all the frames in the sleep section consist of 6 equally. However, node A transmitted a control frame in the second slot of the Data period, and node B transmitted a control signal in the fifth slot. Therefore, the first and last slots became empty slots. Therefore, the Sleep period was changed to 4 slots instead of 6 slots. Since the REF frame is the first control frame, node A can send its data packet at the beginning of each frame. If the node A has more packets to send, the Sleep period can be extended according to the number of packets. With this operational cycle extension capability and the frame slot reduction mechanism, sensor nodes can send more packets quickly.

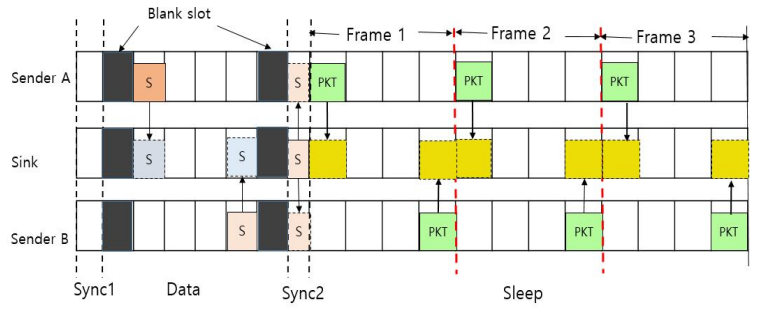

Figure 2: TASA-MAC Protocol scheduling for packet transmission.

\section{PERFORMANCE EVALUATION}

The performance evaluation of TASA-MAC is made through simulation in this section. Here we used the same networking and energy parameters used in [8]. Table 1 shows the network parameters used in the simulations. We also assumed here that all sensors are located in one hop away from the sink node. The network is composed of 30 100 sensors and a sink node.

Table 1: Network Parameters

\begin{tabular}{l|l|l|l}
\hline Parameter & Value & Parameter & Value \\
\hline \hline Bandwidth & 20Kbps & Data pkt size & 50 Bytes \\
\hline$T \_x$ power & $0.5 \mathrm{~W}$ & $T \_s y n c 1$ & $55.2 \mathrm{~ms}$ \\
\hline$R \_x$ Power & $0.5 \mathrm{~W}$ & $T \_$sync 2 & $10 \mathrm{~ms}$ \\
\hline Idle Power & $0.45 \mathrm{~W}$ & $T \_$data & $142.0 \mathrm{~ms}$ \\
\hline Sleep Power & $0.05 \mathrm{~W}$ & $T \_$sleep & $426.0 \mathrm{~ms}$ \\
\hline SIFS & $5 \mathrm{~ms}$ & PION & 14 Bytes \\
\hline DIFS & $10 \mathrm{~ms}$ & SRF, SIF & 14 Bytes \\
\hline RTS,CTS,ACK & $10 \mathrm{Bytes}$ & & \\
\hline
\end{tabular}

And all the data packets from sensors are routed and destined to the sink node, which is located at the center of the network structure. Performance comparison with the related protocol was performed by averaging the results of 10 simulations.

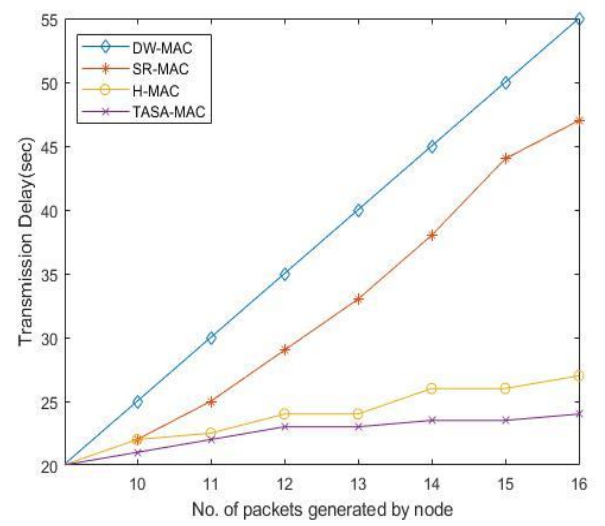

Figure 3: Comparison of the average node's delay 


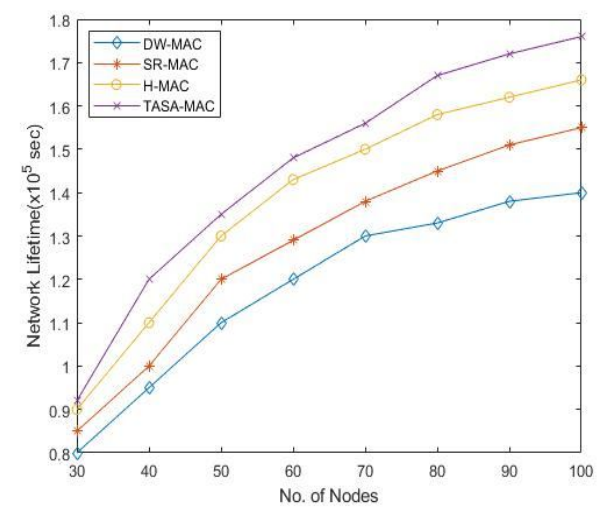

Figure 4: Comparison of the network lifetime

Figure 3 shows the average transmission delay with number nodes in the network. As shown in the figure the TASA-MAC protocol outperforms other related protocols in transmission delay. This is because that senders with higher priority can send bursts data using extended operation period when there is an urgent event. Figure 4 shows the average network lifetime with number nodes in the network.

\section{CONCLUSION}

Data packets generated due to the occurrence of an event such as forest fire, heart attack, wild animal monitoring, and object tracking, generally require priority transmission due to their delay sensitivity. In addition to transmission of these delay-sensitive data packets, the problem of extending the lifetime of the network is very important for WSNs. In this paper, we proposed a traffic aware and super-frame adjustment MAC protocol to solve the above mentioned two problems. The basic idea of this protocol is to transmit burst data packets measured from the event occurrence in a short time without collision. For this, an operational period may be extended according to the traffic amounts to be transmitted. The TASA-MAC protocol reduces the packet transmission delay and prolong the network lifetime in comparison to existing related MAC protocols.

\section{ACKNOWLEDGEMENT}

This research was supported by a 2020 Research Grant from Sangmyung University.

\section{REFERENCES}

1. W. Ye, J. Heidemann, and D. Estrin, An Energy-Efficient MAC Protocol for Wireless Sensor Networks, Proceedings of the 21st Annual Joint Conference of the IEEE Computer and Communications Societies, New York, NY, Vol. 3, 2002, pp. 1567-1576.

2. T. V. Dam, K. Langendoe, An adaptive energy efficient MAC protocol for wireless sensor networks, In Proc. the 1st Int. Conf. Embedded Networked Sensor Systems, (Sensys'03), Los Angeles, California, November 05-07, 2003, pp.171-180. https://doi.org/10.1145/958491.958512

3. S. Du, A. Saha and D. Johnson, RMAC: a routing-enhanced duty-cycle MAC protocol for wireless sensor networks, Proceedings of the 26th Annual IEEE Conference on Computer Communications (INFOCOM 2007), 2007, pp.1478-1486.

4. Y. Sun, S. Du, O. Gurewitz and D. Johnson, DW-MAC: a low latency, energy efficient demand wakeup MAC protocol for wireless sensor networks, Proc. of the Ninth ACM International Symposium on Mobile Ad Hoc Networking and Computing (MobiHoc'08), 2008, pp 53-62.

5. H. Tang, J. Cao, X. Liu, and C. Sun, SR-MAC: A Low Latency MAC Protocol for Multi-Packet Transmission in Wireless Sensor Networks, Journal of Computer Science and Technology, Vol. 28, No. 2, pp. 329-342, March 2013.

https://doi.org/10.1007/s11390-013-1334-4

6. H. Y. Kim, S. C. Kim, A Delay Efficient and Burst Traffics Friendly MAC Protocol in Wireless Sensor Networks, Journal of Korea Multimedia Society (2017), Vol. 20, No. 2, pp. 254-260, February 2017.

7. J. Y. Lee, S. C. Kim, and H.Y. Kim, Energy and Delay Constrained Packet Transmission MAC Protocol for Wireless Sensor Networks, International Journal of Engineering \& Technology, Vol. 7.4.4, pp. 33-34, September 2018.

https://doi.org/10.14419/ijet.v7i4.4.19603

8. S. C. Kim and J. J. Kim, A Superframe Adjustment and Slot Reservation-based MAC Protocol for Wireless Body Area Networks, JP Journal of Heat and Mass Transfer, Special Issue 1, pp.23-27, 2020.

9. Nouf Saeed Alotaibi, An Efficient Technique for Reducing Transmission Energy of Sensor Node to Enhance the Lifetime of the WSN's, International Journal of Advanced Trends in Computer Science and Engineering, Volume 8 No. 2, pp. 108-111, 2019, https://doi.org/10.30534/ijatcse/2019/02822019

10. V. Manikandan and Dr. M. Sivaram, Energy Efficient Dynamic Routing in Wireless Sensor Networks, International Journal of Advanced Trends in Computer Science and Engineering, pp 2189 - 2193, 2019. https://doi.org/10.30534/ijatcse/2019/51852019

11. Yung-Fa Huang, Jong-Shin Chen and Li-Ming Wang, Performance of Transmission Delay and Energy Consumption of Adaptive Listening Mechanism for Wireless Sensor Networks, International Journal of Advanced Trends in Computer Science and Engineering, pp 3215-3220, 2019.

https://doi.org/10.30534/ijatcse/2019/88862019 